\title{
Integration of Drug Use-Results Survey (DUS) Data of a Pharmaceutical Manufacturer
}

\author{
Yasuyuki MATSUSHITA*1, Masahiro SUGIHARA*1, \\ Kaname KAWASUGI*2*3
}

\begin{abstract}
$\langle$ Abstract〉
Objective: Pharmaceutical manufacturers conduct drug use-results surveys (DUSs) for drug reexamination applications in Japan. However, most DUSs are single-cohort studies to confirm drug safety; therefore little information is obtained from a DUS for safety comparison with other drugs. Integrating DUS data is a novel method to compare drug safety profiles among drugs. This study examined the usefulness of integrating DUS data.

Design: Active surveillance

Methods: We integrated DUS data obtained from two DUSs of antihypertensive drugs-a calcium channel blocker (CCB), azelnidipine (Calblock ${ }^{\circledR}$ ), and an angiotensin-receptor blocker (ARB), olmesartan medoxomil $\left(\right.$ Olmetec $\left.^{\circledR}\right)$-with similar study protocols. Both studies were conducted by the same pharmaceutical company. Matching patients using propensity scores, we examined whether the DUS results could be applied to pharmacoepidemiology data resources for hypothesis strengthening. Safety outcomes included vasodilation-related events, which are typical adverse drug reactions (ADRs) to CCB. The incidence of safety outcomes was compared by conditional logistic regression models. Two definitions for safety outcomes were employed: definition 1, physician-reported adverse events; and definition 2, physicianreported ADRs.

Results: In a total of 7196 patients included in the analysis, the propensity-matched patients were well balanced. Most safety outcomes were detected in the CCB group including a significant increase in vasodilation-related events (odds ratio for definition 1 versus ARB group, 1.75; 95\% confidence interval, 1.01-3.03).

Conclusion: Integrated DUS data collated by pharmaceutical manufacturers following clear criteria are potentially useful to commence pharmacoepidemiology studies designed to strengthen hypotheses.
\end{abstract}

Key words: drug use-results survey, propensity score matching, antihypertensive drugs, database

\section{Introduction}

Drug approval during new pharmaceuticals development is based on limited safety information obtained from clinical trials, because the patients enrolled and concomitant drugs used are protocol based. Therefore both pharmaceutical companies and regulatory authorities collect postmarketing drug safety information, assess and analyze those data, and inform physicians about their implications. In Japan, pharmacovigilance for new drugs is conducted based on spontaneous reporting, early postmarketing phase vigilance (EPPV), and a reexamination

${ }^{* 1}$ Clinical Data and Biostatistics Department, Daiichi Sankyo Co., Ltd., Tokyo, Japan

*2 Pharmacovigilance Department, Daiichi Sankyo Co., Ltd., Tokyo, Japan

*3 Department of Allergy and Rheumatology, University of Tokyo School of Medicine, Tokyo, Japan

Address for Correspondence : Y MATSUSHITA, Clinical Data and Biostatistics Department, R\&D Division,

Daiichi Sankyo Co., Ltd., 1-2-58 Hiromachi, Shinagawa-ku, Tokyo 140-8710, Japan 
system as required by the Pharmaceutical Affairs Law. During the reexamination period of 4-10 years postmarketing, a drug use-results survey (DUS), specified drug use survey, and a postmarketing clinical studies may be conducted depending on the drug's characteristics. Japanese pharmaceutical companies reconfirm the quality, effectiveness, and safety of their recently launched drugs based on the results of the DUS, and they report their findings to the Ministry of Health, Labour and Welfare (MHLW) when drugs undergo reexamination ${ }^{12}$.

The DUS is designed to detect or confirm information on the quality, effectiveness, and safety of newly approved drugs and their adverse drug reactions (ADRs) regardless of the disease of the affected patients. An objective of the DUS in many cases is to detect unexpected ADRs and analyze their risk factors. However, most DUSs are single-cohort studies without a control group; therefore safety is not compared among different drugs, and background factors likely to cause ADRs are discussed rather than compared directly.

Postmarketing pharmacovigilance in Japan is conducted mainly by passive surveillance based on spontaneous reports, stimulated reporting by EPPV, and active surveillance by DUS. Since there is no large-scale patient database available in Japan, pharmaceutical companies must conduct primary data collection with each new drug launch. Thus traditional epidemiologic methods corresponding to comparative observational studies in cohort studies or case control studies are not applied unless specifically required by regulatory authorities for new drug approvals.

On the other hand, since many pharmaceutical companies conduct similar postmarketing surveys, an effort has been made to integrate the DUS data. The RAD-AR Council of Japan collects DUS data provided from member companies and has constructed a database to look into the drug safety pharmacoepidemiologically ${ }^{3)}$. However, because this integrated data were composed of collected data by different pharmaceutical companies, data quality and timing of their collection may vary from drug to drug. Several studies were conducted to assess the drug-drug interactions using the database, however, no study to date has compared safety profiles directly between specific drugs included in the database $^{4) 5)}$.

In the present analysis, an attempt was made to investigate whether drug safety information could be improved when one pharmaceutical company integrates DUS data of two separate pharmaceutical compounds from proximal time periods.

\section{Methods}

DUS data on two antihypertensive drugs were analyzed: azelnidipine $\left(\text { Calblock }^{\circledR}\right)^{6}$, a dihydropyridine calcium channel blocker (CCB) launched in Japan in May 2003, and olmesartan medoxomil $\left(\text { Olmetec }^{\circledR}\right)^{7}$, an angiotensin-receptor blocker (ARB) launched in May 2004. DUSs of both Calblock $^{\circledR}$ (AZ DUS) and Olmetec ${ }^{\circledR}$ (OLM DUS) were obtained from single prospective cohort studies conducted in test drug-naïve hypertensive patients in the setting of daily clinical practice. In both studies the observation period was 12 weeks; baseline characteristics of the patients, concomitant drugs administered during the observation period, blood pressure levels, laboratory test values, and adverse events (AEs) and their causal relationship to test agents were surveyed. Generally, in AZ DUS azelnidipine 8 or $16 \mathrm{mg}$ was orally administered once daily whereas in OLM DUS the daily oral dose of olmesartan was 10-20 mg; both were standard dose ranges.

In the AZ DUS and OLM DUS, patient registration was conducted at 1392 institutions between May 2003 and May 2004 and at 1348 institutions between July 2004 and June 2005, respectively. In the two DUSs the incidence of ADRs was $3.5 \%$ among 5146 patients and 3.9\% among 6327 patients assessed for safety, respectively.

These two study protocols were approved by the In-house Ethical Committee of Sankyo (presently Daiichi-Sankyo following merger) and were 
based on the Pharmaceutical Affairs Law in Japan. The protocols were submitted to and approved by the MHLW before study commencement. These studies were carried out in medical institutions registered according to good postmarketing surveillance practice in Japan.

Two definitions for safety outcomes were employed. Definition 1 is physician-reported AEs, whereas definition 2 is physician-reported ADRs. AEs were defined as any undesirable or unintended sign (including abnormal laboratory findings), symptom, or disease temporally associated with the use of test drugs regardless of whether it was considered drug related. ADRs, on the other hand, were defined as safety outcomes of which causal relationship with test drugs could not be ruled out ${ }^{8)}$.

In the present analysis, the typical ADRs of dihydropyridine $\mathrm{CCB}$ were included as safety outcomes. According to the Japanese Society of Hypertension Guidelines for the Management of Hypertension 2009 (JSH 2009) ${ }^{9}$, these include vasodilation-related events such as palpitations, headache, flushing, oedema, gingival growth, and constipation. ADRs were classified using the Preferred Term (PT) in the Medical Dictionary for Regulatory Activities (MedDRA ${ }^{\circledR}$ ) version 12.0. Since clinical symptoms of some PTs in MedDRA $^{\circledR}$ are listed in more than one PT, a number of PTs in the reported AEs/ADRs were integrated by the authors' medical expert (K.K.). For instance flushing, hot flush, and hot feeling were integrated and expressed as flushing whereas face oedema, oedema, and oedema peripheral were integrated and expressed as oedema. Vasodilation-related events included dehydration, vertigo, and positional vertigo in addition to palpitations, headache, flushing, and oedema.

We performed propensity score matching ${ }^{10)}$ of the AZ DUS (CCB group) and OLM DUS (ARB group) based on one-to-one matching without replacement. This method reduces the impact of therapy selection bias in the estimation of the causal treatment effect. The propensity score was estimated using logistic regression on the 32
Drug administration date

\begin{tabular}{|c|c|c|c|}
\hline Jan to Mar & Apr to Jun & Jul to Sep & Oct to Dec \\
\hline CCB group & $\begin{array}{l}\text { One-to-one matching } \\
\text { in the calendar quarter }\end{array}$ \\
\hline ARB group \\
One-to-one \\
matching
\end{tabular}

Figure 1 Propensity score matching with seasonal consideration

baseline covariates described in Table 1 . The estimated propensity score was the predicted probability of receiving olmesartan medoxomil derived from the logistic regression model. Patients in the CCB group who concomitantly took $\mathrm{ARB}$ were expressed as $\mathrm{CCB} / \mathrm{ARB}$ concomitant use while those in the ARB group who concomitantly took $\mathrm{CCB}$ were also expressed as $\mathrm{CCB} / \mathrm{ARB}$ concomitant use. Further, to minimize bias due to the different survey periods, propensity score matching was employed based on the calendar quarter in which test drug administration began (seasonal consideration: Figure 1). Each quarterly period was matched without consideration of the specific date when drug administration began, because the registration periods are different in the two surveys.

Matched data were extracted by matching AZ DUS and OLM DUS on the logit of the propensity score using calipers of width equal to 0.2 of the standard deviation (0.2 SD) of the propensity score logit. A greedy, nearest-neighbor matching algorithm was employed to form pairs of azelnidipine-treated and olmesartan medoxomiltreated patients ${ }^{1112)}$. We then compared the balance in baseline characteristics between matched patients in the $\mathrm{CCB}$ and $\mathrm{ARB}$ groups based on standardized differences ${ }^{12) 13)}$ and distributions of propensity scores.

The incidence of an interested safety outcome $j, P_{j k}$, was analyzed by the following conditional logistic regression model stratified by $\mathrm{K}$ matched 
pairs:

$$
\operatorname{logit} P_{j k}=\gamma_{j k}+\beta_{j} X,
$$

where $\gamma_{j k}$ is the matched pair-specific regression parameter at stratum $k(k=1, \ldots, \mathrm{K}), \beta_{j}$ is the regression parameter for the safety outcome $j$, and $X$ is the treatment indicator variable $(0, A R B$ group; 1, CCB group). Each matched pair was based on one-to-one matching and consisted of one patient from the CCB group and one patient from the OLM group. The univariate logistic regression model as a crude analysis was also applied to the total cohort.

Two sensitivity analyses were performed: one without seasonal consideration of the data, and an alternative reanalysis in which the condition of greedy matching was altered to the matching on the propensity score using calipers set a width of 0.03 , because Austin et $\mathrm{al}^{12)}$ reported that matching using calipers at a width of $0.2 \mathrm{SD}$ of the logit of the propensity score and the use of calipers at a width 0.02 and 0.03 tended to have a superior performance for estimating treatment effects.

Statistical significance level was considered as a 2 -sided $P$-value $<5 \%$. All statistical analyses were performed using SAS version 9.2 (SAS Institute, Cary, NC, USA) software.

\section{Results}

Of 11473 cases in the total cohort (AZ DUS, $n$ $=5146$; OLM DUS, $n=6327$ ), propensity scorematched cases in the $\mathrm{CCB}$ and $\mathrm{ARB}$ groups totaled 3598 each $(69.9 \%$ and $56.9 \%$ of the two groups, respectively). The actual value of calipers was 0.168 set at a width of $0.2 \mathrm{SD}$ of the logit of the propensity score.

Baseline patient characteristics before and after adjustment are shown in Table 1. Before adjustment, there were more women in whom systolic/diastolic blood pressure (SBP/DBP) was $2 / 1 \mathrm{mmHg}$ higher, on average, in the CCB group. On the other hand, more patients in the ARB group were previously or concurrently taking other antihypertensive drugs. Furthermore, $\mathrm{CCB} / \mathrm{ARB}$ concomitant use was significantly different between the two groups. In all, 8 of 32 variables had a standardized difference $>10 \%$. The distribution of the propensity score in the total cohort is shown in Figure 2. The propensity score of the ARB group showed two-peaked. The most influencing factor for the propensity score was the previous use of CCB medication. The propensity scores differed very much by the presence of the previous use of CCB medication, and this resulted in a two-peaked distribution in the propensity scores.

After propensity score matching, the imbalance between the two groups was largely improved and the standardized difference in all variables was $<10 \%$. The distribution of the propensity score in matched patients is shown in Figure 3. The distributions were well balanced between the two groups of matched patients; no evidence was obtained that the two distributions in the matched patients were different $(P=$ 0.6793; Kolmogorov-Smirnov test).

The analyzed results of safety outcomes are summarized in Tables 2 and 3. Odds ratios (ORs) for incidence of palpitations, headache, flushing, and oedema in the CCB versus ARB group were all $>1.0$ in matched patients. ORs for vasodilation-related events of definitions 1 and 2 were 1.75 (95\% confidence interval [CI], 1.01-3.03; $P=0.046)$ and 2.50 (95\% CI, $1.28-4.88 ; P=$ 0.007 ), respectively, indicating that vasodilationrelated events associated with CCB were significantly more common than with ARB. Furthermore, ORs in matched patients tended to be larger than in the total cohort. The absolute risk difference of vasodilation-related events between the groups was $0.42 \%$ for definition 1 and $0.50 \%$ for definition 2. However, no gingival growth was reported in either group, and no constipation was reported in the CCB group. The likelihood ratio chi-square test statistics in the conditional logistic models for vasodilation-related events of definitions 1 and 2 were $\chi^{2}=4.143(P=0.042)$ and $\chi^{2}=7.970 \quad(P=0.005)$, respectively.

The results of sensitivity analysis without seasonal consideration of the data are shown in Table 4. Propensity score-matched cases in the 
CCB and ARB groups were 4322 each. Compared with the season matched data, the ORs for the sensitivity analysis were close to 1 . In the analysis in which the caliper was changed from $0.2 \mathrm{SD}$ of the propensity score logit to 0.03 of the propensity score, matched cases in the $\mathrm{CCB}$ and ARB groups were 3590 each, which is close to the number of cases obtained in the main analysis; the results were also similar (data not shown).

\section{Discussion}

The optimal pharmacovigilance method to address a specific situation may vary depending on the drug product, the indication, the target population, and the issue to be addressed ${ }^{14)}$. Strom et al. $^{15)}$ proposed three stages such as hypothesis generating, hypothesis strengthening, and hypothesis testing as strategies to confirm drug safety using different pharmacoepidemiologic data resources. In Western countries, hypothesis-strengthening studies and hypothesistesting studies are conducted using various databases such as the General Practice Research Database (GPRD) and Health Databases in Saskatchewan ${ }^{16)}$. Although Japan does not have databases that correspond to these databases, the Japan Pharmaceutical Manufacturers Association (JPMA) has proposed that utilization of integrated DUS data may be an effective avenue toward hypothesis generating and hypothesis strengthening ${ }^{17)}$. According to the JPMA, risk magnitude can be compared among similar drugs based on database information collected on comparable drugs, when a certain signal suggesting risk is detected according to spontaneous reports. Thus we investigated whether information to strengthen the signal can be obtained when DUS data on two different drugs with similar indications and efficacy are integrated.

Of 11473 integrated cases, there were 7198 propensity-matched cases, indicating that $62.7 \%$ of all cases were integrated. Following propensity score matching, imbalances between the groups were largely improved, and the standardized difference of all variables was $<10 \%$. Further, the distribution of the propensity scores nearly overlapped after matching and no evidence was found to indicate a different distribution. Thus matching was deemed successful.

After adjustment, the factors with a notable change were baseline blood pressure, prior concomitant $\mathrm{CCB}$ therapy, and proportion of $\mathrm{CCB} / \mathrm{ARB}$ concomitant use when the investigation was started. When considering seasonal variations, the ORs for vasodilation-related events tended to increase in matched patients. Vasodilation-related AEs caused by CCBs are a well known risk associated with taking these medications ${ }^{18)}$. In the present study, although a significantly higher risk of vasodilation-related AEs and palpitations was detected, there was only a slightly increased risk of other AEs. This is considered to be related to the observation that the therapeutic effects of azelnidipine appear slowly and therefore vasodilation-related AEs caused by this agent are less than those associated with other $\mathrm{CCBs}^{1920)}$.

When DUS data on two or more drugs developed by different companies are integrated, the descriptors (PTs) of AEs may be different due to different study variables and survey methods. Thus drug safety data must be integrated in a manner that ensures similar survey variables and data collection and management methods are used and that targeted survey groups closely match. All data analyzed in the present study fulfilled these criteria.

Two definitions for capturing safety outcomes were used in the present study; AEs and ADRs. Unlike medical insurance claims databases and electronic health record systems, the integrated DUS data we analyzed were unique because of the inclusion of the decisions of the attending physician. Among vasodilation-related events, the ORs of AEs and ADRs were 1.75 and 2.50, respectively. The absolute risk difference was $0.42 \%$ and $0.50 \%$, respectively. Thus both the $\mathrm{OR}$ and risk difference of AEs were lower than those of ADRs. This finding may be due to information bias in that vasodilation-related events of CCBs are well known and therefore may be more frequently reported. It may be 


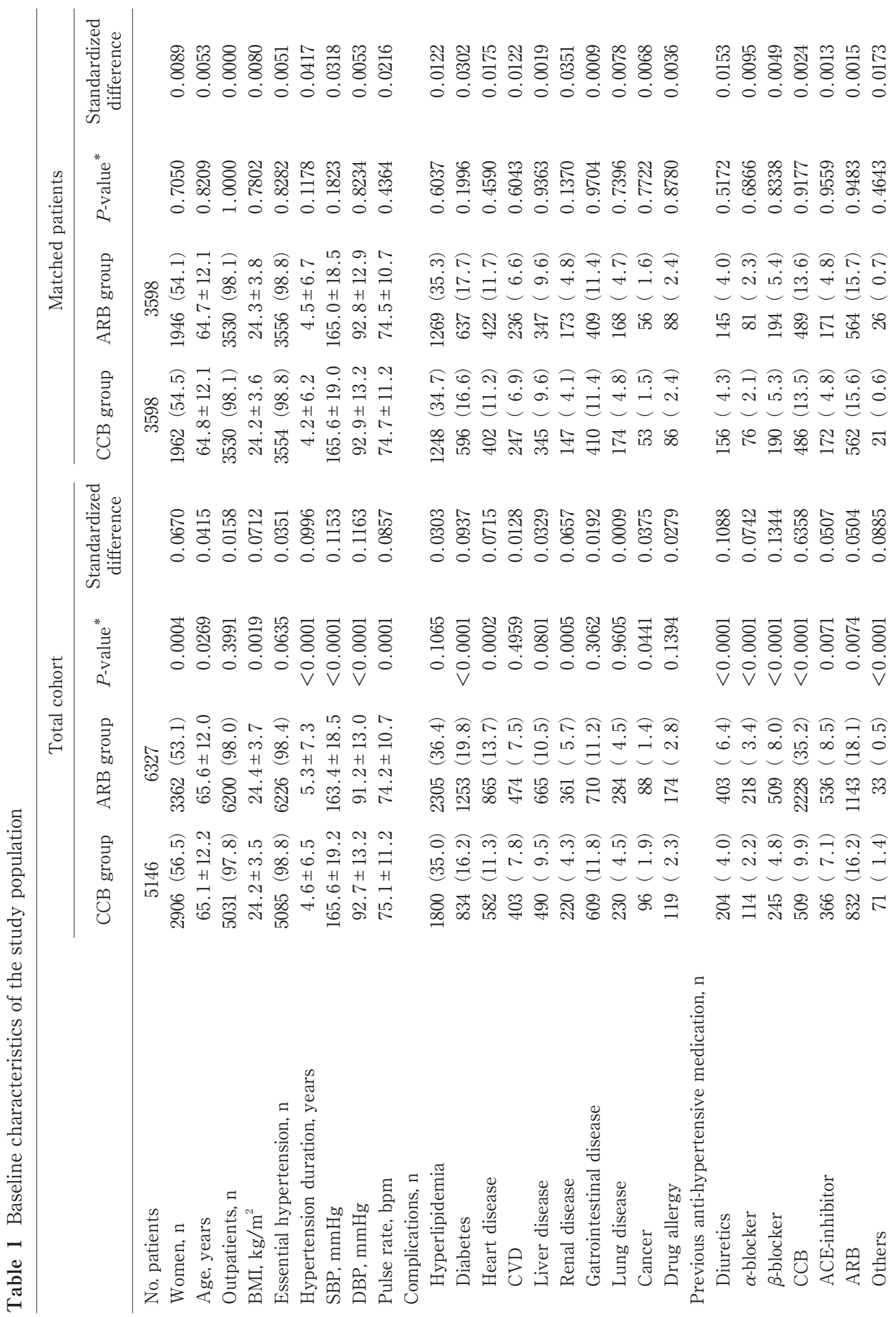


薬撤疫学 Jpn J Pharmacoepidemiol, 15(2) Dec 2010：67

ஓ융 융 ్ㅠㅇ

$\because 00000$

ஜ

000000

ศิ

H N 以 $\smile \smile \smile=\smile$

员

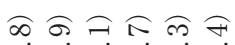

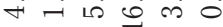

오용 \&

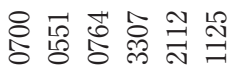

$\circ 00000$

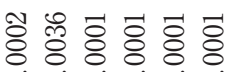

$\dot{0} \dot{0} \dot{0} \dot{V} \dot{V} \dot{V} \dot{V}$

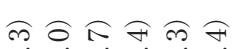

$\dot{\varphi} \dot{\varphi} \dot{\vec{m}} \dot{\mathrm{j}}$

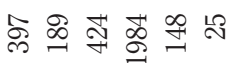

즈응

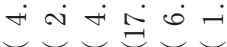

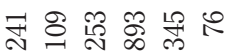

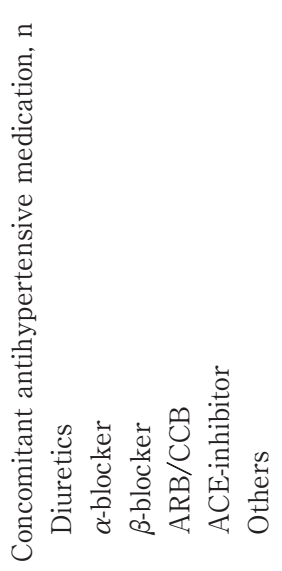

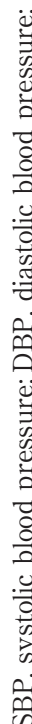
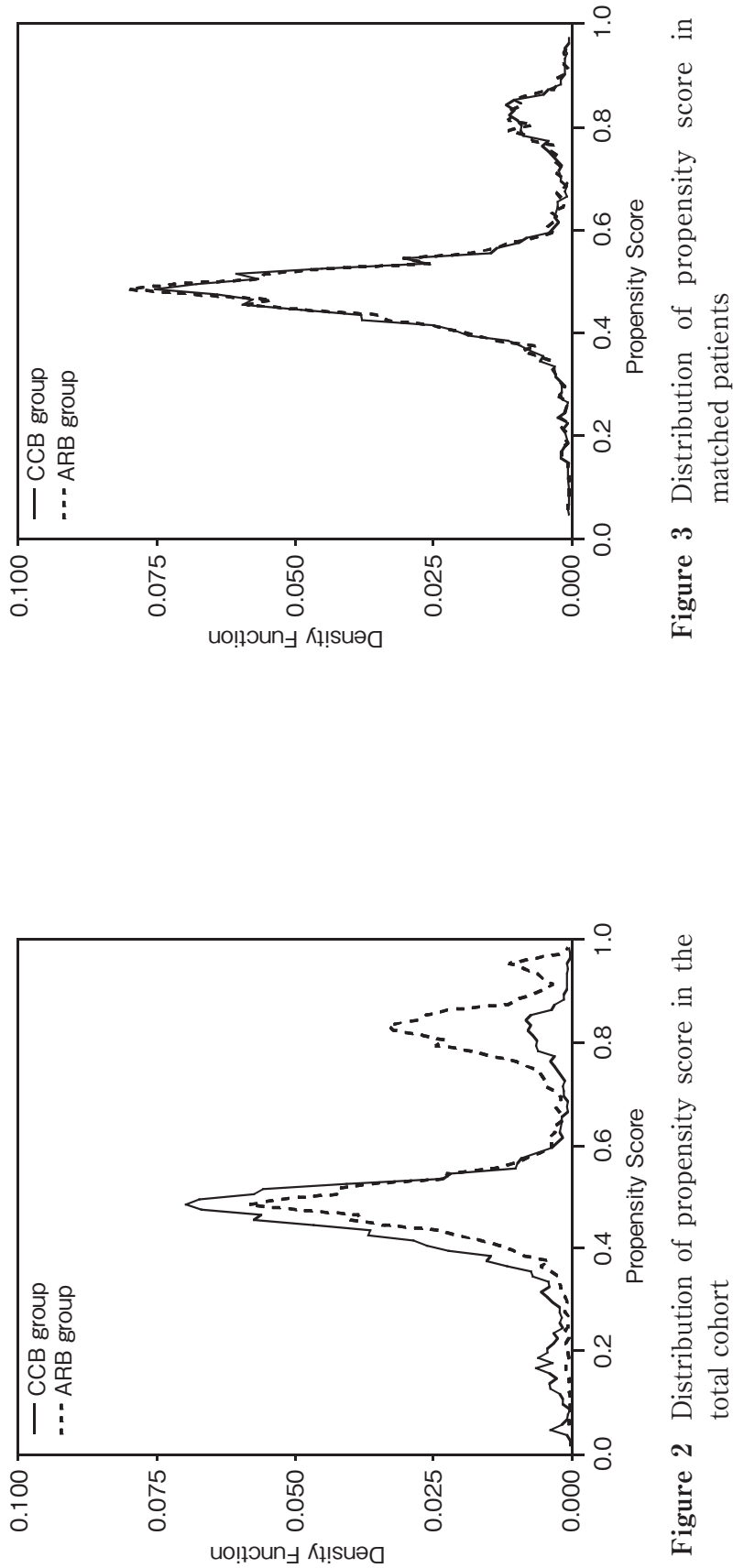


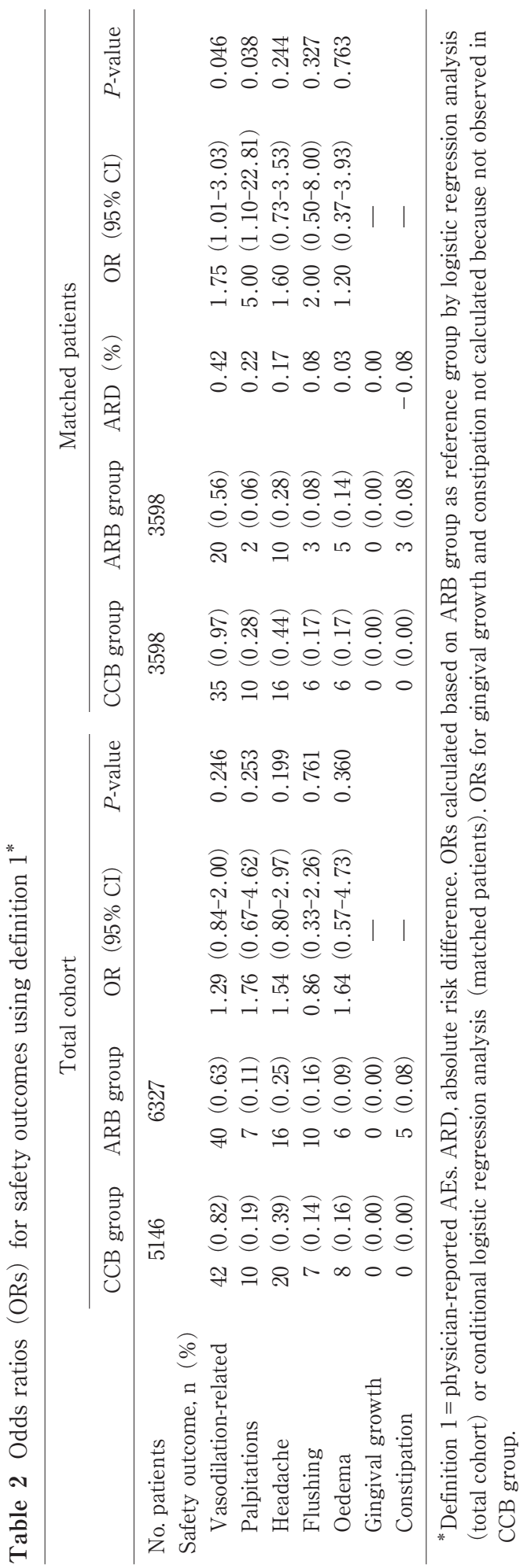

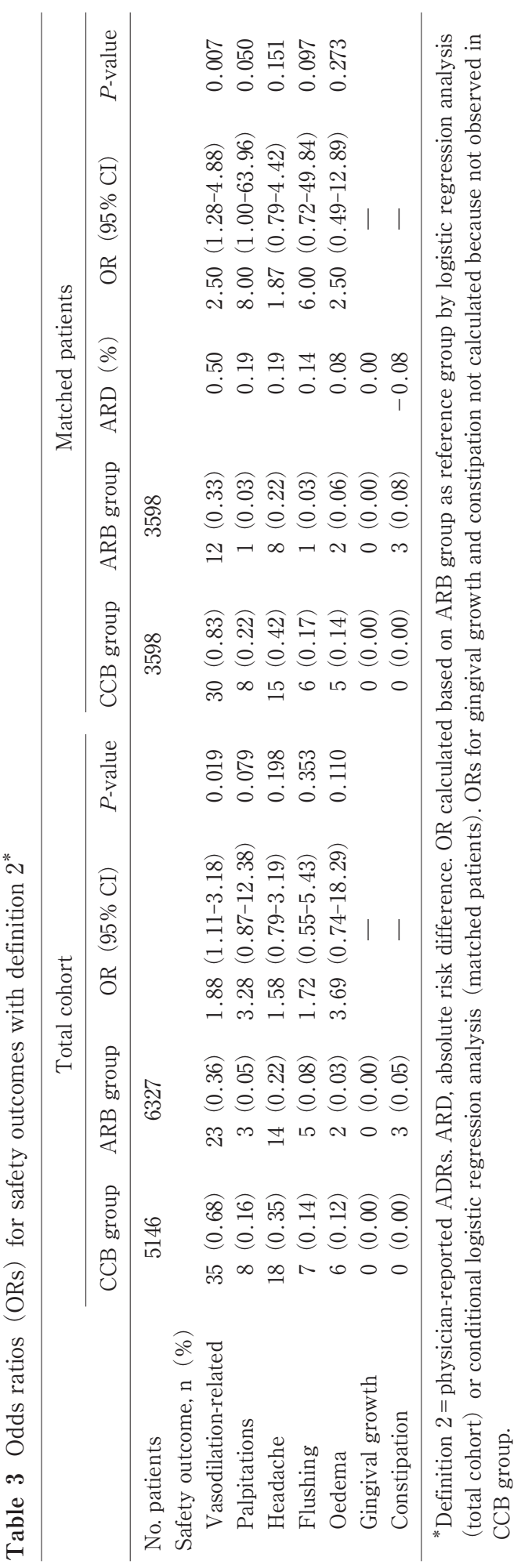




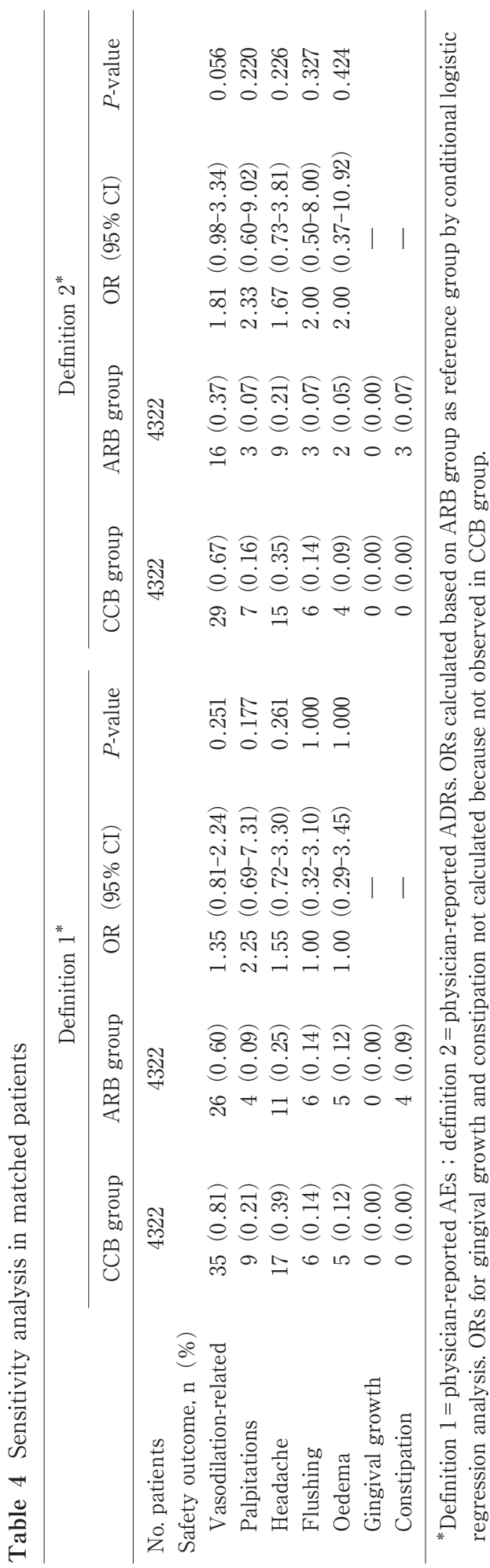

important to confirm whether the direction of the results is identical in both definitions.

In this study MedDRA ${ }^{\circledR}$ PTs were used to encode AEs and ADRs. However, some PTs were integrated. According to Pearson et $\mathrm{al}^{21}{ }^{21}$ it is important to investigate drug safety data using High Level Terms (HLT) and Standardized MedDRA Query (SMQ). Although we tried to use HLT, some events were not adequately integrated. For instance, flushing was defined as flushing, hot flush, and feeling hot in the present study. However, in HLT, flushing and hot flush correspond to peripheral vascular disorders. On the other hand, our definition of oedema was identical with that described in HLT. Thus it seems useful to use HLT and SMQ judiciously depending on the events examined.

The limitations in the present study are insufficient matching factors and small sample size. Factors used in the propensity score matching were 32 individual baseline characteristics of the patients. However, unmeasured confounding factors may also exist. Ishiguro et al. ${ }^{5)}$ previously assessed the effects of nonsteroidal anti-inflammatory drugs on antihypertensive drugs by matching patients in each DUS using the antihypertensive drug database. Matching patients in each DUS is a valid method to consider the differences of the investigation methods in each DUS; however, we did not match patients in each DUS in our study. Yet, since the present survey was carried out by a single company and the investigational methods were quite similar in both DUS, integration of the data was determined to be possible. Furthermore, since the study period of these two surveys was one year apart, the present investigation used season matching, not time matching. The blood pressure of patients with essential hypertension has a seasonal variation with higher pressures in winter than in the summer ${ }^{22)}$. According to the sensitivity analysis, some bias could be regulated by season matching. Also since the medical environment changes little over one year, any differences attributable to the year of the survey are considered small. 
Although the ORs for headache, flushing, and oedema in the CCB versus ARB group were $>1$, the differences were not statistically significant. Although this is considered primarily due to characteristics of the drugs themselves, the small sample size may have contributed at least in part to the finding.

In the sample size calculation for cohort studies, our study size would yield a power of $>0.90$ with a 2 -tailed $\alpha$ value of 0.05 to detect a relative risk of 2.0 for an $\mathrm{AE}$ with a $1.0 \%$ occurrence as background rate. This study size also could detect a relative risk of 2.5 for an $\mathrm{AE}$ with a background rate of $0.5 \%$, and relative risk of 3.0 for an $\mathrm{AE}$ with a $0.3 \%$ background rate ${ }^{23)}$.

According to our results, integrating data obtained from DUS seems useful to strengthen hypotheses delivered from spontaneous reporting, although the data quality is not sufficient for hypothesis testing. In addition, since many baseline characteristic factors of the patients were investigated in the DUS in the present study, analysis using the propensity score method was considered appropriate.

The US Food and Drug Administration began its Sentinel initiative in May 2008 and announced that they will construct a novel postmarketing monitoring system employing a database of 100 million patients by $2012^{24)}$. In Europe, the EUADR project has begun to allow signal detection using multinational clinical information databases ${ }^{25}$. In Japan, the Pharmaceuticals and Medical Devices Agency announced a new initiative to computerize information on adverse drug reactions, such as drug use-result survey, and by 2014 to build databases to provide for the use of centralized electronic data for the development of safety measures ${ }^{26)}$. The methods used in the present study could be applied to analyze safety information in the DUSs.

Rezaltas ${ }^{\circledR}$, the combination tablet comprising azelnidipine and olmesartan, was recently approved in Japan and a DUS is underway ${ }^{27}$. Compared with patients on single-drug regimens, those prescribed combination drug preparations are at inherently higher risk. Thus it may be difficult to obtain sufficient drug safety information on combination preparations from simple comparisons with each drug when used alone. Therefore, it is likely that our integrated analysis of DUS data on azelnidipine and olmesartan will prove useful to predict the clinical safety profile of Rezaltas ${ }^{\circledR}$, even though the investigation time is different.

\section{Conclusion}

Integrated DUS data collated by pharmaceutical manufacturers following clear criteria are potentially useful to commence pharmacoepidemiology studies designed to strengthen hypotheses.

\section{Acknowledgments}

The authors would like to express their sincere gratitude to the many physicians who cooperated in the present DUSs. The authors would like to thank the two anonymous reviewers whose comments greatly improved this article.

\section{Conflict of interest}

All authors are employed by Daiichi Sankyo Co. Ltd. No sources of funding were used to assist in the preparation of this study.

\section{References}

1) Post marketing surveillance. In: Overview of PMS System. Tokyo : Jiho, 2008 : 31-49 (in Japanese).

2) Tanaka K, Morita Y, Kawabe E, Kubota K. Drug use investigation (DUI) and prescription-event monitoring in Japan (J-PEM). Pharmacoepidemiol Drug Saf. $2001 ; 10: 653-8$.

3) Fujita T, Miura Y, Mayama T. A pilot study to build a database on seven anti-hypertensive drugs. Pharmacoepidemiol Drug Saf. 2005 ; 14 : 41-6.

4) Yoshida M, Matsumoto T, Suzuki T, Kitamura S, Mayama T. Effect of concomitant treatment with a CYP3A4 inhibitor and a calcium channel blocker. Pharmacoepidemiol Drug Saf. 2008 ; 17 : 70-5.

5) Ishiguro C, Fujita T, Omori T, Fujii Y, Mayama T, Sato T. Assessing the effects of non-steroidal antiinflammatory drugs on antihypertensive drug therapy using post-marketing surveillance database. J Epidemiol. 2008 ; 18 : 119-24.

6) Kitai T, Murata E, Uehara Y, et al. Use-result surveillance of azelnidipine (Calblock ${ }^{\circledR}$ Tab).J Clin Ther Med. $2005 ; 21: 511-27$ (in Japanese). 
7) Saito I, Kushiro T, Yamakawa M, et al. Drug use result survey of Olmetec ${ }^{\circledR}$ tablets (olmesartan medoxomil).J Clin Ther Med. $2006 ; 22: 873-93$ (in Japanese).

8) ICH-E2A-Expert Working Group. Clinical safety data management: definitions and standards for expedited reporting. [http://www.ich.org/LOB/ media/MEDIA436.pdf-accessed 2010-10-18].

9) The Japanese Society of Hypertension Guidelines for the Management of Hypertension (JSH 2009). Hypertens Res. 2009 ; 32 : 3-107.

10) Rosenbaum PR, Rubin DB. The central role of the propensity score in observational studies for causal effects. Biometrika. $1983 ; 70$ : 41-55.

11) Rosenbaum PR, Rubin DB. Constructing a control group using multivariate matched sampling methods that incorporate the propensity score. Am Stat. $1985 ; 39: 33-8$.

12) Austin PC. Some methods of propensity-score matching had superior performance to others: results of an empirical investigation and Monte Carlo simulations. Biometr J. 2009 ; 51 : 171-84.

13) Austin PC. A critical appraisal of propensity-score matching in the medical literature between 1996 and 2003. Stat Med. $2008 ; 27: 2037-49$.

14) ICH-E2E-Expert Working Group. Pharmacovigilance planning. [http://www.ich.org/LOB/media/ MEDIA1195.pdf-accessed 2010-10-18]

15) Strom BL. How should one perform pharmacoepidemiology study? Choosing among the available alternatives. In: Pharmacoepidemiology, 4th ed. New York : John Wiley \& Sons, 2005.

16) Wood L, Martinez C. The general practice research database : role in pharmacovigilance. Drug Saf. $2004 ; 27: 871-81$.

17) Post-marketing Surveillance Subcommittee, Drug Evaluation Committee, Japan Pharmaceutical Manufactures Association. Potential of drug use-results survey data for pharmacoepidemiological research. Jpn J Pharmacoepidemiol. 2009 ; 14 : 53-9 (in Japanese).

18) Kubota K, Pearce GL, Inman WHW. Vasodilationrelated adverse events in diltiazem and dihydropyridine calcium antagonists studied by prescriptionevent monitoring. Eur J Clin Pharmacol. 1995 ; 48 : $1-7$.
薬剂疫学 Jpn J Pharmacoepidemiol, 15(2) Dec 2010 : 71

19) Yoram Y, Ahuva L. Azelnidipine (CS-905), a novel dihydropyridine calcium channel blocker with gradual onset and prolonged duration of action. Cardiovasc Drug Rev. 1995 ; 13 : 137-48.

20) Yoshinaga $\mathrm{K}$, Iimura $\mathrm{O}$, Abe $\mathrm{K}$, et al. A multicenter double-blind comparison study of CS-905 (azelnidipine) with nitrendipine in patients with essential hypertension. J Clin Ther Med. 2000; 16:671-739 (in Japanese).

21) Pearson RK, Hauben M, Goldsmith DI, et al. Influence of the MedDRA ${ }^{\circledR}$ hierarchy on pharmacovigilance data mining results. Int J Med Inform. $2009 ; 78:$ e97-e103.

22) Hata T, Ogihara T, Maruyama A, et al. The seasonal variation of blood pressure in patients with essential hypertension. Clin Exp Hypertens A. 1982 ; 4 : 34154.

23) Schlesslman JJ. Sample size requirements in cohort and case-control studies of disease. Am J Epidemiol. $1974 ; 99: 381-4$.

24) Platt R, Wilson M, Chan KA, Benner JS, Marchibroda J, McClellan M. The new Sentinel network-improving the evidence of medical-product safety. N Engl J Med. 2009 ; $361: 645-7$.

25) Trifiro G, Pariente A, Coloma PM, et al. ; EU-ADR Group. Data mining on electronic health record databases for signal detection in pharmacovigilance: which events to monitor? Pharmacoepidemiol Drug Saf. $2009 ; 18: 1176-84$.

26) Authorization No. 0331002 issued by the Pharmaceutical and Food Safety Bureau, Ministry of Health, Labour and Welfare. Mid-term Plan of the Pharmaceuticals and Medical Devices Agency. [http://www.pmda.go.jp/english/about/pdf/Sec ond_Mid-term_Plan.pdf-accessed 2010-10-18].

27) Pharmaceuticals and Medical Devices Agency. Review reports of new product applications. [http:// www.info.pmda.go.jp/shinyaku/g100116/43057400_ 22200A MX00244_A100_2.pdf-accessed 2010-1018] (in Japanese).

Manuscript received October 20, 2010 revised December 23, 2010 accepted January 7, 2011 